PROCEEDINGS OF THE

AMERICAN MATHEMATICAL SOCIETY

Volume 140, Number 4, April 2012, Pages 1343-1348

S 0002-9939(2011)11023-2

Article electronically published on August 3, 2011

\title{
THE PRINCIPAL INVERSE OF THE GAMMA FUNCTION
}

\author{
MITSURU UCHIYAMA
}

(Communicated by Richard Rochberg)

\begin{abstract}
Let $\Gamma(x)$ be the gamma function in the real axis and $\alpha$ the maximal zero of $\Gamma^{\prime}(x)$. We call the inverse function of $\left.\Gamma(x)\right|_{(\alpha, \infty)}$ the principal inverse and denote it by $\Gamma^{-1}(x)$. We show that $\Gamma^{-1}(x)$ has the holomorphic extension $\Gamma^{-1}(z)$ to $\mathbf{C} \backslash(-\infty, \Gamma(\alpha)$ ], which maps the upper half-plane into itself, namely a Pick function, and that $\Gamma\left(\Gamma^{-1}(z)\right)=z$ on $\mathbf{C} \backslash(-\infty, \Gamma(\alpha)]$.
\end{abstract}

\section{INTRODUCTION}

The Euler form of the gamma function $\Gamma(x)$ is given by

$$
\Gamma(x)=\int_{0}^{\infty} e^{-t} t^{x-1} d t
$$

for $x>0$. The Weierstrass form

$$
\frac{1}{\Gamma(x)}=x e^{\gamma x} \prod_{n=1}^{\infty}\left(1+\frac{x}{n}\right) e^{-\frac{x}{n}}
$$

extends it to $\mathbf{R} \backslash\{0,-1,-2, \cdots\}$, where $\gamma$ is the Euler constant defined by

$$
\gamma=\lim _{n \rightarrow \infty}\left(1+\frac{1}{2}+\cdots+\frac{1}{n}-\log n\right)=0.57721 \cdots .
$$

From this it follows that

$$
\begin{aligned}
\log \Gamma(x) & =-\log x-\gamma x+\sum_{n=1}^{\infty}\left(\frac{x}{n}-\log \left(1+\frac{x}{n}\right)\right), \\
\frac{\Gamma^{\prime}(x)}{\Gamma(x)} & =-\gamma+\sum_{n=0}^{\infty}\left(\frac{1}{n+1}-\frac{1}{n+x}\right) .
\end{aligned}
$$

This is called the psi function or digamma function. It is clear that $\Gamma(1)=\Gamma(2)=$ $1, \Gamma^{\prime}(1)=-\gamma, \Gamma^{\prime}(2)=-\gamma+1$. Denote the unique zero in $(0, \infty)$ of $\Gamma^{\prime}(x)$ by $\alpha$. It is known that $\alpha=1.4616 \cdots$ and $\Gamma(\alpha)=0.8856 \cdots$. We call the inverse function of the restriction of $\Gamma(x)$ to $(\alpha, \infty)$ the principal inverse function and denote it by $\Gamma^{-1}$. $\Gamma^{-1}(x)$ is an increasing and concave function defined on $(\Gamma(\alpha), \infty)$. (1.1) guarantees that $\Gamma(x)$ has the holomorphic extension which is a meromorphic function with poles at non-positive integers and (1.3) holds there. Let $\Pi_{+}$and $\Pi_{-}$be respectively the

Received by the editors November 18, 2010 and, in revised form, January 4, 2011.

2010 Mathematics Subject Classification. Primary 33B15; Secondary 26A48, 47A63.

Key words and phrases. Gamma function, Pick function, Nevanlinna function, Loewner kernel function.

The author was supported in part by (JSPS) KAKENHI 21540181.

(C)2011 American Mathematical Society 1343

Reverts to public domain 28 years from publication 
open upper half-plane and the open lower half-plane. A holomorphic function defined on $\Pi_{+}$is called a Pick function or Nevanlinna function if it maps $\Pi_{+}$into itself. From (1.3) it follows that $\Gamma^{\prime}(z)$ does not vanish on $\mathbf{C} \backslash(-\infty, \alpha]$; in fact, $\Gamma^{\prime}(z) / \Gamma(z)$ is a Pick function. Hence for each point $\omega_{0} \in \Gamma(\mathbf{C} \backslash(-\infty, \alpha])$ there is a local inverse of $\Gamma(z)$ in a neighborhood of $\omega_{0}$.

Some other Pick functions related to the gamma function were investigated in [1] and [2].

The main objective of this paper is to show

Theorem 1. The principal inverse $\Gamma^{-1}(x)$ of $\Gamma(x)$ has the holomorphic extension $\Gamma^{-1}(z)$ to $\mathbf{C} \backslash(-\infty, \Gamma(\alpha)]$, which satisfies

(i) $\Gamma^{-1}\left(\Pi_{+}\right) \subset \Pi_{+}$and $\Gamma^{-1}\left(\Pi_{-}\right) \subset \Pi_{-}$,

(ii) $\Gamma^{-1}(z)$ is univalent,

(iii) $\Gamma\left(\Gamma^{-1}(z)\right)=z$ for $z \in \mathbf{C} \backslash(-\infty, \Gamma(\alpha)]$.

We remark that (iii) implies that $D:=\Gamma^{-1}(\mathbf{C} \backslash(-\infty, \Gamma(\alpha)])$ is a domain including $(\alpha, \infty)$ and $\Gamma(D)=\mathbf{C} \backslash(-\infty, \Gamma(\alpha)]$, and $\Gamma^{-1}(\Gamma(z))=z$ for $z \in D$.

To prove Theorem 1 we use the theory of kernel functions. Let $K(x, y)$ be a real continuous function defined on $I \times I$ and suppose $K(x, y)=K(y, x)$. Then $K(x, y)$ is said to be a positive semidefinite (abbreviated to p.s.d.) kernel function on an interval $I \times I$ if

$$
\iint_{I \times I} K(x, y) \phi(x) \phi(y) d x d y \geqq 0
$$

for every real continuous function $\phi$ with compact support in $I$. In this case, (1.4) holds for complex-valued functions $\phi(x)$ as well, provided we take the complex conjugate of $\phi(y)$. It is clear that $K(x, y)$ is p.s.d. if and only if for each $n$ and for all $n$ points $x_{i} \in I$, the $n \times n$ matrices

$$
\left(K\left(x_{i}, x_{j}\right)\right)_{i, j=1}^{n}
$$

are positive semidefinite matrices. Suppose $K(x, y) \geqq 0$ for every $x, y$ in $I$. Then $K(x, y)$ is said to be infinitely divisible if $K(x, y)^{a}$ is p.s.d. for every $a>0 . K(x, y)$ is said to be conditionally (or almost) positive semidefinite (abbreviated to c.p.s.d.) on $I$ if (1.4) holds for every continuous function $\phi$ on $I$ such that the support of $\phi$ is compact and the integral of $\phi$ over $I$ vanishes. One can see that $K(x, y)$ is c.p.s.d. if and only if

$$
\sum_{i, j=1}^{n} K\left(x_{i}, x_{j}\right) z_{i} \overline{z_{j}} \geqq 0
$$

for each $n$, for all $n$ points $x_{i} \in I$ and for $n$ complex numbers $z_{i}$ with $\sum_{i=1}^{n} z_{i}=0$. Let $f(x)$ be a $C^{1}$-function on $I$. Then the Löwner kernel function is defined by

$$
K_{f}(x, y)=\left\{\begin{array}{cc}
\frac{f(x)-f(y)}{x-y} & (x \neq y), \\
f^{\prime}(x) & (x=y) .
\end{array}\right.
$$

The following excellent theorem is due to Löwner [7] (also see Koranyi [6] and [8]).

Theorem A. Let $f(x)$ be a $C^{1}$-function on $I$. Then the Löwner kernel function $K_{f}(x, y)$ is p.s.d. if and only if $f(x)$ has a holomorphic extension $f(z)$ to $\Pi_{+}$and it is a Pick function. 


\section{Proof of Theorem 1}

We begin with a simple fact which follows from Theorem A because $\log x$ defined on $(0, \infty)$ extends to a Pick function.

\section{Lemma 2.}

$$
K_{1}(x, y):=\left\{\begin{array}{cc}
\frac{\log x-\log y}{x-y} & (x \neq y) \\
\frac{1}{x} & (x=y)
\end{array}\right.
$$

is p.s.d. on $(0, \infty) \times(0, \infty)$.

Lemma 3. Let $K_{2}(x, y)$ be the function defined on $(0, \infty) \times(0, \infty)$ by

$$
K_{2}(x, y):=\left\{\begin{array}{cc}
\frac{\log \Gamma(x)-\log \Gamma(y)}{x-y} & (x \neq y) \\
\frac{\Gamma^{\prime}(x)}{\Gamma(x)} & (x=y) .
\end{array}\right.
$$

Then $-K_{2}(x, y)$ is c.p.s.d. on $(0, \infty)$.

Proof. Suppose that the support of $\phi(x)$ is included in $[m, M]$ with $m>0$ and $\int_{m}^{M} \phi(x) d x=0$. From (1.2) it follows that $-K_{2}(x, y)=K_{1}(x, y)+\gamma-K_{g}(x, y)$, where $K_{g}$ is a Löwner kernel function of $g$ defined by

$$
g(x)=\sum_{k=1}^{\infty}\left(\frac{x}{k}-\log \left(1+\frac{x}{k}\right)\right) .
$$

Since $K_{1}(x, y)$ is p.s.d. and $\int_{0}^{\infty} \int_{0}^{\infty} \gamma \phi(x) \phi(y) d x d y=0$, we have only to show that $-K_{g}(x, y)$ is c.p.s.d. Put

$$
g_{n}(x)=\sum_{k=1}^{n}\left(\frac{x}{k}-\log \left(1+\frac{x}{k}\right)\right) .
$$

Then

$$
g_{n}^{\prime}(x)=\sum_{k=1}^{n} \frac{x}{k(k+x)}
$$

converges uniformly to $\sum_{k=1}^{\infty} \frac{x}{k(k+x)}=g^{\prime}(x)$ on $[0, M]$. The sequence of Löwner kernel functions $K_{g_{n}}(x, y)$ converges uniformly to $K_{g}(x, y)$ on $[0, M] \times[0, M]$; indeed,

$$
K_{g_{n}}(x, y)-K_{g}(x, y)=\left\{\begin{array}{cc}
\frac{1}{x-y} \int_{y}^{x}\left(g_{n}^{\prime}(t)-g^{\prime}(t)\right) d t & (x \neq y) \\
g_{n}^{\prime}(x)-g^{\prime}(x) & (x=y) .
\end{array}\right.
$$

Since

$$
-K_{g_{n}}(x, y)=\sum_{k=1}^{n}\left(-\frac{1}{k}+\frac{1}{k} K_{1}\left(1+\frac{x}{k}, 1+\frac{y}{k}\right)\right)
$$

is c.p.s.d., so is $-K_{g}(x, y)$.

Note that a similar proof is given in [3], where it is proved that $\Gamma(z)^{c}$ is the Mellin transform of a positive measure on $(0, \infty)$ for each $c>0$.

The following is known ([8], p. 152; 9 and [10]), but for completeness we give a proof. 
Lemma 4. Let $K(x, y)>0$ for $x, y \in I$. If $-K(x, y)$ is c.p.s.d. on $I \times I$, then the reciprocal function $\frac{1}{K(x, y)}$ is infinitely divisible there.

Proof. Take $x_{i} \in I(i=1,2, \cdots, n)$ and put $a_{i j}=K\left(x_{i}, x_{j}\right)$. Define $b_{i j}$ by

$$
b_{i j}=a_{i j}-a_{i n}-a_{n j}+a_{n n} \quad(1 \leqq i, j \leqq n) .
$$

Since $-a_{i j}=-K\left(x_{i}, x_{j}\right)$ satisfies (1.5), the matrix $\left(-b_{i j}\right)$ is positive semidefinite (see p. 134 of [8] or p. 458 of [11]). By Schur's theorem, the matrix $\left(e^{-b_{i j}}\right)$ is positive semidefinite too.

$$
e^{-a_{i j}}=e^{-a_{i n}+\frac{a_{n n}}{2}} e^{-b_{i j}} e^{-a_{n j}+\frac{a_{n n}}{2}}
$$

implies that the matrix $\left(\exp \left(-a_{i j}\right)\right)$ is p.s.d. as well. Thus we have shown that $\exp (-K(x, y))$ is p.s.d. We note that $\exp (-t K(x, y))$ is also p.s.d. for $t>0$ since $-t K(x, y)$ is c.p.s.d. By making use of

$$
\Gamma(a)=k^{a} \int_{0}^{\infty} e^{-k t} t^{a-1} d t \quad(a>0)
$$

we get

$$
K(x, y)^{-a}=\frac{1}{\Gamma(a)} \int_{0}^{\infty} \exp (-t K(x, y)) t^{a-1} d t,
$$

which is p.s.d. This implies that $1 / K(x, y)$ is infinitely divisible.

Lemma 5. Let $K_{3}(x, y)$ be the kernel function defined on $(\alpha, \infty) \times(\alpha, \infty)$ by

$$
K_{3}(x, y)=\left\{\begin{array}{cc}
\frac{x-y}{\Gamma(x)-\Gamma(y)} & (x \neq y) \\
\frac{1}{\Gamma^{\prime}(x)} & (x=y) .
\end{array}\right.
$$

Then $K_{3}(x, y)$ is p.s.d.

Proof. Let $K_{1}(x, y)$ and $K_{2}(x, y)$ be the kernel functions defined in Lemma 2 and Lemma 3, respectively. Since $K_{1}(x, y)$ is p.s.d. on $(0, \infty) \times(0, \infty)$ and since $\Gamma(x)$ is differentiable and increasing on $(\alpha, \infty)$,

$$
K_{1}(\Gamma(x), \Gamma(y))=\left\{\begin{array}{cc}
\frac{\log \Gamma(x)-\log \Gamma(y)}{\Gamma(x)-\Gamma(y)} & (x \neq y) \\
\frac{1}{\Gamma(x)} & (x=y)
\end{array}\right.
$$

is p.s.d. on $(\alpha, \infty) \times(\alpha, \infty)$. Since $\Gamma(x)$ is increasing on $(\alpha, \infty), K_{2}(x, y)>0$ for $(x, y) \in(\alpha, \infty) \times(\alpha, \infty)$. By Lemma 3 and Lemma 4, $\frac{1}{K_{2}(x, y)}$ is not only p.s.d. but also infinitely divisible. Thus the Schur product

$$
K_{3}(x, y)=K_{1}(\Gamma(x), \Gamma(y)) \cdot \frac{1}{K_{2}(x, y)}
$$

is p.s.d. on $(\alpha, \infty) \times(\alpha, \infty)$ too.

Proof of Theorem 1. The Löwner kernel $K_{\Gamma^{-1}}(x, y)$ defined on $(\Gamma(\alpha), \infty)$ $\times(\Gamma(\alpha), \infty)$ by

$$
K_{\Gamma^{-1}}(x, y)=\left\{\begin{array}{cc}
\frac{\Gamma^{-1}(x)-\Gamma^{-1}(y)}{x-y} & (x \neq y) \\
\left(\Gamma^{-1}\right)^{\prime}(x) & (x=y)
\end{array}\right.
$$


coincides with $K_{3}\left(\Gamma^{-1}(x), \Gamma^{-1}(y)\right)$, which is p.s.d. Thus by Theorem $\mathrm{A}, \Gamma^{-1}(x)$ has the holomorphic extension $\Gamma^{-1}(z)$ onto $\Pi_{+}$, which is a Pick function. By reflection, $\Gamma^{-1}(x)$ also has a holomorphic extension to $\Pi_{-}$and the range is in it. We thus get (i). $\Gamma\left(\Gamma^{-1}(z)\right)$ is thus holomorphic on the simply connected domain $\mathbf{C} \backslash(-\infty, \Gamma(\alpha)]$, and $\Gamma\left(\Gamma^{-1}(x)\right)=x$ for $\Gamma(\alpha)<x<\infty$. By the uniqueness theorem, $\Gamma\left(\Gamma^{-1}(z)\right)=z$ for $z \in \mathbf{C} \backslash(-\infty, \Gamma(\alpha)]$. This means (iii), which clearly yields (ii).

In the proof of Lemma 4 we saw that if $-K(x, y)$ is c.p.s.d., then $e^{-K(x, y)}$ is infinitely divisible. For $K_{2}(x, y)$ in Lemma 3 .

$$
e^{-K_{2}(x, y)}=\left\{\begin{array}{cc}
\left(\frac{\Gamma(y)}{\Gamma(x)}\right)^{\frac{1}{x-y}} & (x \neq y) \\
e^{-\frac{\Gamma^{\prime}(x)}{\Gamma(x)}} & (x=y)
\end{array}\right.
$$

is infinitely divisible. Since $\Gamma(x+1)=x \Gamma(x)$,

$$
\frac{\Gamma^{\prime}(1)}{\Gamma(1)}=-\gamma, \frac{\Gamma^{\prime}(m+1)}{\Gamma(m+1)}=-\gamma+1+\cdots+\frac{1}{m}, \frac{\Gamma(n)}{\Gamma(m)}=\frac{(n-1) !}{(m-1) !} .
$$

The following $(n+1) \times(n+1)$ matrix is therefore not only p.s.d. but also infinitely divisible:

$$
\left(e^{-K_{2}(i, j)}\right)=\left(\begin{array}{cccccc}
e^{\gamma} & \left(\frac{1 !}{1 !}\right)^{-1} & (2 !)^{-\frac{1}{2}} & (3 !)^{-\frac{1}{3}} & \cdots & (n !)^{-\frac{1}{n}} \\
\left(\frac{1 !}{1 !}\right)^{-1} & e^{\gamma-1} & \left(\frac{2 !}{1 !}\right)^{-1} & \left(\frac{3 !}{1 !}\right)^{-\frac{1}{2}} & \cdots & \left(\frac{n !}{1 !}\right)^{-\frac{1}{n-1}} \\
(2 !)^{-\frac{1}{2}} & \left(\frac{2 !}{1 !}\right)^{-1} & e^{\gamma-1-\frac{1}{2}} & \left(\frac{3 !}{2 !}\right)^{-1} & \cdots & \left(\frac{n !}{2 !}\right)^{-\frac{1}{n-2}} \\
(3 !)^{-\frac{1}{3}} & \left(\frac{3 !}{1 !}\right)^{-\frac{1}{2}} & \left(\frac{3 !}{2 !}\right)^{-1} & e^{\gamma-1-\frac{1}{2}-\frac{1}{3}} & \cdots & \left(\frac{n !}{3 !}\right)^{-\frac{1}{n-3}} \\
\vdots & \vdots & \vdots & \vdots & \ddots & \vdots \\
(n !)^{-\frac{1}{n}} & \left(\frac{n !}{1 !}\right)^{-\frac{1}{n-1}} & \left(\frac{n !}{2 !}\right)^{-\frac{1}{n-2}} & \left(\frac{n !}{3 !}\right)^{-\frac{1}{n-3}} & \cdots & e^{\gamma-1-\frac{1}{2}-\cdots-\frac{1}{n}}
\end{array}\right) .
$$

Since $\Gamma^{-1}(z)$ is holomorphic on $\mathbf{C} \backslash(-\infty, \Gamma(\alpha)]$ and a Pick function, by Herglotz's theorem $\Gamma^{-1}(x)$ has the following integral representation with the Borel measure $\mu(t)([4,[8],[12]):$

\section{Corollary 6.}

$$
\Gamma^{-1}(x)=a+b x+\int_{-\infty}^{\Gamma(\alpha)}\left(\frac{1}{x-t}-\frac{t}{t^{2}+1}\right) d \mu(t),
$$

where $\int_{-\infty}^{\Gamma(\alpha)} \frac{1}{t^{2}+1} d \mu(t)<\infty$, and $a, b$ are real numbers and $b \geqq 0$.

Question. How can we extend the inverse function of the restricted function of $\Gamma(x)$ to $(0, \alpha)$ ?

\section{ACKNOWLEDGEMENTS}

The author thanks Prof. A. Uchiyama for his helpful advice. He is also grateful to the referee for pointing out several simple mistakes and giving him valuable comments.

\section{REFERENCES}

[1] H. Alzer and C. Berg, Some classes of completely monotonic functions II, Ramanujan J. 11(2006)225-248. MR2267677 (2007k:33001)

[2] C. Berg and H. L. Pedersen, Pick functions related to the gamma function, Rocky Mountain J. of Math. 32(2002)507-525. MR1934903 (2004h:33007) 
[3] C. Berg, On powers of Stieltjes moment sequences, I, J. Theoretical Probability 18(2005)871889. MR2289936 (2008i:44008)

[4] R. Bhatia, Matrix Analysis, Springer, 1996. MR.1477662 (98i:15003)

[5] R. Bahtia and T. Sano, Loewner matrices and operator convexity, Math. Ann. 344(2009)703716. MR2501306 (2010g:47037)

[6] A. Koranyi, On a theorem of Löwner and its connections with resolvents of selfadjoint transformations, Acta Sci. Math. 17(1956)63-70. MR0082656 (18:588c)

[7] K. Löwner, Über monotone Matrixfunctionen, Math. Z. 38(1934)177-216. MR1545446

[8] W. F. Donoghue, Monotone Matrix Functions and Analytic Continuation, Springer-Verlag, 1974. MR0486556 (58:6279)

[9] C. H. Fitzgerald, On analytic continuation to a Schlicht function, Proc. Amer. Math. Soc. 18(1967)788-792. MR0219712 (36:2791)

[10] R. A. Horn, Schlicht mapping and infinitely divisible kernels, Pacific J. of Math. 38(1971)423430. MR0310208 (46:9310)

[11] R. A. Horn and C. R. Johnson, Topics in Matrix Analysis, Cambridge University Press, 1991. MR.1091716 (92e:15003)

[12] M. Rosenblum and J. Rovnyak, Hardy Classes and Operator Theory, Oxford Univ. Press, 1985. MR822228 (87e:47001)

[13] M. Uchiyama, Operator monotone functions, positive definite kernels and majorization, Proc. Amer. Math. Soc. 138(2010)3985-3996. MR2679620

Department of Mathematics, Interdisciplinary Faculty of Science and Engineering, Shimane University, Matsue City, Shimane 690-8504, Japan

E-mail address: uchiyama@riko.shimane-u.ac.jp 